\title{
Frontières
}

\section{La représentation du rapport à la mort dans Le Nécrophile de Gabrielle Wittkop}

\section{Julie Delorme}

Volume 18, numéro 1, automne 2005

Hélas, célébrer la mort!

URI : https://id.erudit.org/iderudit/1074310ar

DOI : https://doi.org/10.7202/1074310ar

Aller au sommaire du numéro

Éditeur(s)

Université du Québec à Montréal

ISSN

1180-3479 (imprimé)

1916-0976 (numérique)

Découvrir la revue

Citer cet article

Delorme, J. (2005). La représentation du rapport à la mort dans Le Nécrophile de Gabrielle Wittkop. Frontières, 18(1), 21-26. https://doi.org/10.7202/1074310ar
Résumé de l'article

Dans la représentation du rapport à la mort, le stéréotype joue un rôle crucial. Ce sont souvent des images préconstruites qui sont associées à l'idée que l'on se forge de la mort. La dépouille devient l'objet d'une exclusion sociale. Or, il s'avère que Le Nécrophile de Gabrielle Wittkop fait appel à des stratégies discursives et à une écriture qui peuvent être considérées comme une subversion du discours établi. En s'appropriant les restes d'autrui, le personnage du nécrophile transgresse à la fois les deux plus grands tabous de l'Occident : la sexualité et la mort. C'est d'ailleurs en ce sens que le protagoniste célèbre la mort. Plutôt que de céder à la répulsion face à la dépouille, le nécrophile la fête à partir d'un rapport rituel. 


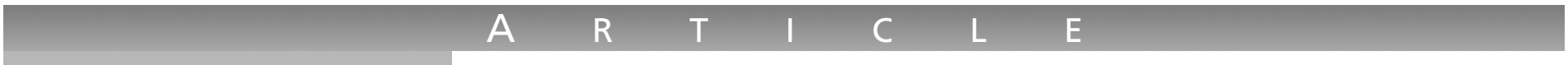 \\ Résumé \\ Dans la représentation du rapport à la mort, le stéréotype joue un rôle crucial. Ce sont souvent des images préconstrui- tes qui sont associées à l'idée que l'on se forge de la mort. La dépouille devient I'objet d'une exclusion sociale. Or, il s'avère que Le Nécrophile de Gabrielle \\ LA REPRÉSENTATION DU RAPPORT À LA MORT DANS LE NÉCROPHILE DE GABRIELLE WITTKOP} Wittkop fait appel à des stratégies discursives et à une écriture qui peuvent être considérées comme une subversion du discours établi. En s'appropriant les restes d'autrui, le personnage du nécrophile transgresse à la fois les deux plus grands tabous de l'Occident: la sexualité et la mort. C'est d'ailleurs en ce sens que le protagoniste célèbre la mort. Plutôt que de céder à la répulsion face à la dépouille, le nécrophile la fête à partir d'un rapport rituel.

Mots clés: Gabrielle Wittkop sexualité - mort - littérature.

\section{Abstract}

The stereotype plays a crucial part in the representation of death. In fact, pre-constructed images are most often associated to the idea that we construct of death. The mortal remains become an object of social exclusion. "Le Nécrophile" of Gabrielle Wittkop appeals to discursive strategies and writing approach that can be considered as a subversion of the established discourse. In trying to appropriate the remains of the other, the necrophilic character transgresses the two major occidental taboos: sexuality and death. It is in this way that the protagonist celebrates death. Instead of being repulsed by the cadaver, the Necrophiliac celebrates it through a ritual relationship.

Keywords: Gabrielle Wittkop sexuality - death - literature.

\author{
Julie Delorme, \\ Département des lettres françaises, \\ Université d'Ottawa.
}

\section{POUR UNE APPROCHE DIACHRONIQUE DU RAPPORT À LA MORT}

L'art pariétal, dont les grottes de Lascaux constituent à la fois le témoignage et l'héritage par excellence, est une inspiration de la peur de la mort. Les nombreux hiéroglyphes qui y sont dessinés représentent des situations d'angoisse dans lesquelles l'homme se confronte à l'animal. La pensée primitive démonisait la mort dans la mesure où elle y voyait un agent menaçant, voire haineux à l'égard du vivant. Même si l'on sait aujourd'hui que la mort d'autrui n'est pas «contagieuse», elle continue, dans le cadre de nos sociétés contemporaines, de se voir attribuer la valeur de tabou.

La violence sous-jacente à la mort est probablement ce qui la fait encore craindre. Hegel, de même que Freud, souligne que «la mort est ce qu'il y a de plus terrible ${ }^{1}$ ». Il n'y a de mort qui soit paisible. Tous les organismes sont prédisposés à connaître une mort qui soit violente. Inspiré par la pensée hégélienne, Georges Bataille en vient également à croire qu'il ne peut exister de force qui ne soit plus grande que celle-là: «[L'état] le plus violent pour nous est la mort qui, précisément, nous arrache à l'obstination que nous avons de voir durer l'être discontinu que nous sommes» (Bataille, 2001, p. 23). On a affaire ici à l'un des traits qui distinguent l'homme de l'animal: cette capacité à reconnaître qu'il va un jour s'éteindre. Afin d'échapper temporairement à la mort, l'homme s'invente des stratégies dans lesquelles il croit pouvoir oublier le réel. C'est souvent dans une sorte de théâtre intérieur qu'il choisit d'établir son existence. Bref, c'est parce que la mort fait peur qu'il la refoule.

Malgré la peur qu'elle inspire et tous les interdits qui planent sur elle, la société postmoderne est attirée par tout ce qui touche à la mort, ce qui est tout à fait paradoxal. Il suffit, par exemple, de feuilleter un journal pour se rendre compte que la représentation du rapport à la mort y occupe une place prépondérante. On y trouve autant d'images qui meublent, pour ne pas dire qui envahissent le quotidien des Occidentaux ${ }^{2}$. C'est dans ce contexte que l'on peut penser notre société actuelle comme une société nécrophile, voire «imagophage» (Castillo Durante, 2004, p. 118). Elle opère comme un véritable sarcophage qui, plutôt que de chair en décomposition, se nourrit d'images de corps morts, lesquelles à force d'être répétées et maintes fois recyclées finissent, comme le stéréotype, par s'user. Pour le dire d'une façon un peu brutale, la société contemporaine se nourrit des dépouilles d'autrui. Elle cherche à récupérer les restes que, paradoxalement, la société de consommation laisse pour compte. Or, l'Occident est à cheval sur deux paliers à la fois. D'une part, il s'ancre dans un système itératif où le stéréotype et la copie sont au cœur d'une philosophie de recyclage alors que, d'autre part, il consomme sans regard au gaspillage qu'il engendre. Aussi contradictoire que cela paraisse, c'est comme si on vivait dans un monde où les logiques inhérentes à la nécrophilie - récupération - et à la prostitution - consommation (Delorme, 2005) seraient simultanément à l'œuvre. 


\section{ÉROS ET THANATOS:}

\section{UNE DIALECTIQUE PARADOXALE}

Que se passe-t-il quand la mort et la sexualité (les deux tabous majeurs selon Bataille) s'interpénètrent? Tout porte à croire que cette fusion donne lieu à un interdit paré d'une double contrainte: la nécrophilie. Celle-ci appert comme une union "perverse» puisqu'elle force le sujet à penser le «pire» dans sa représentation la plus subversive où Éros et Thanatos s'entrechoquent: la preuve que la mort et la sexualité ne sont peut-être pas si étrangères l'une pour l'autre.

L'érotisme, tel que le définit Le petit Robert, relève d'un "goût marqué pour le plaisir sexuel, $[\ldots]$ caractère de ce qui a l'amour physique pour thème [...]» (Robert, 2004, p. 938). Il faut néanmoins savoir qu'au-delà de l'acception du dictionnaire, la présence du préfixe "éros», tiré de la mythologie grecque, désigne la divinité de l'Amour ${ }^{3}$. C'est d'ailleurs de ce mythe antique que Freud s'inspire pour élaborer sa théorie sur les personnalités: "En psychanalyse, éros est synonyme d'instinct de vie. C'est l'énergie instinctuelle primordiale qui veille à la préservation et à la perpétuation de chacun» (Morin et Bouchard, 1997, p. 22). Le père de la psychanalyse met en parallèle le concept de libido à celui d'éros. Pour lui, la libido correspond à l'énergie psychique associée à l'instinct de vie.

Le couple «Éros et Thanatos» témoigne ainsi de la dualité freudienne qui voit l'individu comme un être de contradiction. Si Georges Bataille dans un énoncé qui peut d'abord sembler curieux stipule que l'érotisme «est l'approbation de la vie jusque dans la mort» (Bataille, 2001, p. 17), nous pourrions presque en dire autant de la nécrophilie qui, elle, se veut un concept paradoxal. C'est-à-dire que pour assouvir son désir de vie (éros), le sujet nécrophile fait appel à ses pulsions de mort (thanatos). Eu égard au fait que le personnage de Wittkop considère le mort comme un vivant en lui attribuant presque toutes les caractéristiques de l'espèce, il s'avère doublement transgressif. Chez le sujet nécrophile, éros et thanatos ne doivent donc pas être compris comme deux concepts distincts tels que Freud le souhaite mais plutôt comme une seule et même entité.

Acceptant cette prémisse, il nous importe de voir comment s'articule cette dialectique dans le cadre littéraire. Mais plus spécifiquement, notre analyse vise à montrer d'emblée quelles sont les stratégies discursives qui sous-tendent la représentation du rapport à la mort dans le roman de Gabrielle Wittkop, Le Nécrophile. On peut d'ores et déjà poser l'hypothèse selon laquelle ce texte rend compte de la perméabilité de la frontière entre la vie et la mort dans une écriture qui parviendrait à rompre

\section{MALGRÉ LA PEUR QU'ELLE INSPIRE}

\section{ET TOUS LES INTERDITS QUI PLANENT SUR ELLE,}

\section{LA SOCIÉTÉ POSTMODERNE EST ATTIRÉE}

\section{PAR TOUT CE QUI TOUCHE À LA MORT,}

\section{CE QUI EST TOUT À FAIT PARADOXAL.}

En contrepartie, la mort est définie dans Le petit Robert selon une approche essentiellement scientifique et clinique du terme désignant «la cessation définitive de la vie» (Robert, 2004, p. 1674) d'un sujet. Mais ce serait faire preuve de dogmatisme que d'affirmer que cet état ontologique se résume à cette simple définition, ignorant le paradoxe dans lequel la mort s'allie à la vie. En effet, par «opposition à éros, thanatos désigne les pulsions de mort qui pourraient provoquer une tendance constante à retourner à un état premier d'inanition » (Morin et Bouchard, 1997, p. 23). Freud précise cependant que «thanatos» ne doit pas être systématiquement associé à l'instinct de mort au sens fatal du terme mais bien en l'expression d'une certaine destructivité ou agressivité inhérente chez l'homme (Morin et Bouchard, 1997, p. 17). avec le discours stéréotypé que l'on tient à l'endroit des morts et de leur sépulture.

\section{LE NÉCROPHILE}

\section{UNE PAROLE «MÉTATOPIQUE»}

La représentation met en place des modalités à partir desquelles la littérature opère. Il arrive que la parole littéraire récupère des images qu'elle s'évertue à raviver par diverses stratégies discursives, notamment celle de la transgression. L'écriture de Gabrielle Wittkop cherche sans détour à remettre en question le tabou de la mort tel qu'il apparaît en Occident en mettant en scène un personnage nécrophile mais, en même temps, elle subvertit le stéréotype dont cet interdit est l'objet. C'est en quoi elle pourrait être qualifiée de «métatopique» (Castillo Durante, 1994).
La transgression n'est pas, comme le dit Georges Bataille, «la négation de l'interdit mais elle le dépasse et le complète» (Bataille, 2001, p. 71). Le Nécrophile traite presque exclusivement de cette capacité à outrepasser les limites de l'interdit. D'un point de vue phénoménologique, la transgression liée à la mort s'explique par ce principe où le cadavre n'est pas simplement considéré comme un objet inerte mais bien comme une personne vivante. Le texte serait donc en train de peindre le caractère éternel des choses, des êtres et, c'est en cela que réside également la complexité du nécrophile. Certes, dans le roman, on assiste à une certaine décomposition de la matière organique mais, dans une perspective purement philosophique, c'est comme si le nécrophile, dans son activité, empêchait les choses d'avoir une finitude. Cela rejoint le concept scientifique voulant que toute matière, quelle qu'elle soit, n'ait ni début ni fin ${ }^{4}$. À certains égards, le personnage du nécrophile se refuse ainsi à l'exclusion des morts telle qu'elle opère en Occident. Donc, en traitant les cadavres comme des êtres vivants, on lève le voile, non pas sur un seul tabou - celui de la mort - mais sur une kyrielle d'interdits qui, nous le verrons, tendent à se chevaucher, voire à s'emboîter comme dans une sorte de mise en abyme.

D'abord, le nécrophile viole dans son acte l'un des interdits liés à la mort. Dans cette pratique, il y a transgression de la dernière volonté de l'être, ce qui rend cette conduite illégale et donc condamnable par la loi: «On parle du sexe sous toutes ses formes, sauf une. La nécrophilie n'est ni tolérée des gouvernements ni approuvée des jeunesses contestataires » (Wittkop, 2001, p. 49). En effet, dans ce roman, le nécrophile ne respecte pas ses victimes car il les expulse de leur sépulture. Ce n'est toutefois pas sans crainte d'être surpris en flagrant délit qu'il s'adonne à cette activité. Le protagoniste éprouve souvent la sensation qu'on l'épie, redoutant les individus qui l'entourent; la preuve qu'il est conscient de sa marginalité: "J'ai souvent l'impression qu'on m'observe, qu'on me guette. Surtout les personnes de service, femmes de ménage, concierges, commerçants du quartier. Et les flics, bien sûr. Surtout les flics»(Wittkop, 2001, p. 49). Il ne faut toutefois pas croire que tout s'arrête une fois ce seuil franchi. Le narrateur évolue en suivant une sorte de crescendo convergeant vers le «pire».

\section{LE FANTASME \\ DE LA MORT D'AUTRUI COMME MODE DE SUBVERSION}

En plus de manquer de respect envers le défunt, le protagoniste fantasme sur les cadavres ou plutôt sur les vivants qu'il 
souhaiterait morts. Cela constitue une transgression dans la mesure où cette "production de l'imagination par laquelle le moi cherche à échapper à l'emprise de la réalité» (Robert, 2004, p. 1035) éveille la libido $^{5} \mathrm{du}$ personnage. Par ces représentations mentales, on voit clairement la préférence marquée du nécrophile pour les morts. Dès l'incipit, le lecteur se rend compte de ce désir inhérent au personnage. Le soir même où l'enfant découvre les plaisirs de la masturbation, il apprend le décès de sa mère. Pourrait-on voir dans cette coïncidence de la découverte de la mort et de l'onanisme une condition de possibilité de l'émergence ultérieure de la nécrophilie chez le personnage?

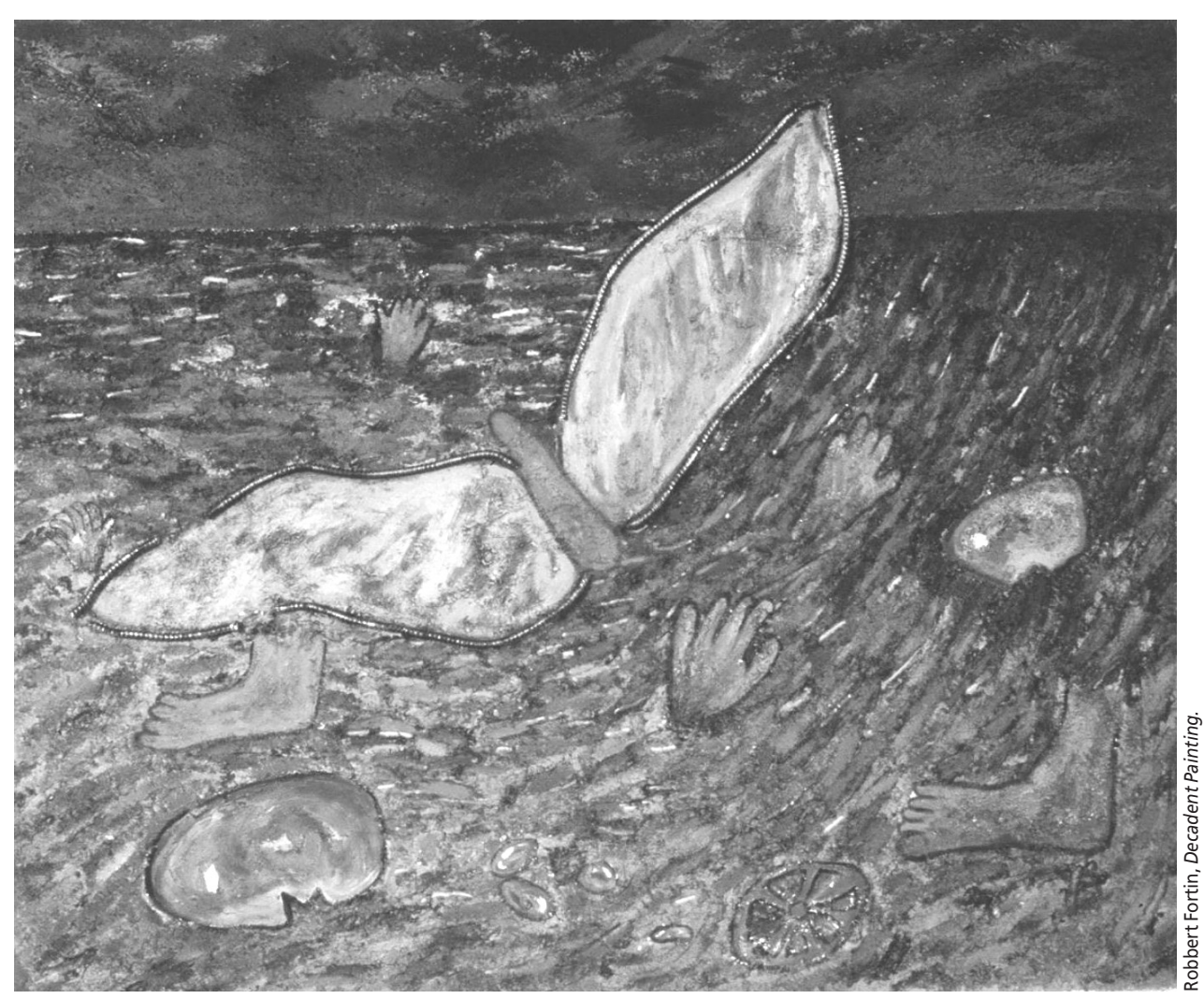

J'avais tout juste huit ans. Un soir de novembre [...] on m'avait laissé seul dans ma chambre [...] Pour me distraire et me consoler, j'entrepris de déboutonner ma petite culotte. J'y trouvai cette chose chaude et douce qui toujours me tenait compagnie. Je ne sais plus comment ma main découvrit les mouvements qu'il fallait, mais je fus soudain saisi dans un vortex de délices [...] Je m'étonnai infiniment [...] de sentir mes proportions se modifier [...] J'activai mes mouvements et ma volupté s'accrut encore mais, alors même qu'une vague qui me semblait née au fond de mes entrailles, paraissait vouloir me submerger et me soulever au-dessus de moi-même [...] la porte s'ouvrit [...] ma grand-mère se tenait sur le seuil et son trouble était si grand qu'elle ne remarqua pas l'état où je me trouvais. "Mon pauvre enfant!

Ta maman est morte.»

(Wittkop, 2001, p. 15-16)

L'inclination du narrateur envers la nécrophilie est quelque chose qui relève d'une connaissance intrinsèque; personne n'a appris au personnage à se comporter de la sorte. Aussi, ce passage montre-t-il la tendance qu'ont les adultes à occulter aux enfants certaines réalités comme la mort, un tabou qui se trouve ici annulé par le discours sans grand euphémisme de la grand-mère: «Ta maman est morte.» En dernière analyse, le narrateur du roman de sexuelles après quoi le nécrophile est tenté de lui répondre, non sans un soupçon de sarcasme: "J'aimerais beaucoup vos yeux révulsés, vos lèvres muettes, votre sexe glacial, si seulement vous étiez mort. Malheureusement, vous avez le très mauvais goût d'être en vie [...]»(Wittkop, 2001, p. 51). Conscient qu'il ne peut avouer la vérité, le narrateur affirme ne pas être libre pour ne pas avoir à justifier son attirance envers les morts, sachant pertinemment que la nécrophilie n'est pas chose courante parce que, justement, elle ressortit à une transgression sociale. C'est donc à une sorte d'autocensure que doit s'assujettir le personnage du nécrophile.

La représentation psychique que se forge le narrateur du personnage de Gabrielle est un fantasme qui illustre de manière précise le registre imaginaire dans lequel il évolue:

Je ne puis voir une jolie femme ou un homme agréable sans immédiatement souhaiter qu'ils fussent morts.

Jadis, [...] je le souhaitais même avec passion, avec fureur. Il s'agissait d'une voisine [...] que j'apercevais presque tous les jours. Bien que la désirant, jamais l'idée ne me serait venue de seulement toucher sa main. J'attendais, je voulais sa mort et cette mort devenait pour moi le pôle autour duquel gravitaient toutes mes pensées [...] Alors, je me dépeignais pendant des heures tous les dangers et tous les modes de décès qui pouvaient frapper Gabrielle. J'aimais me la représenter sur son lit de mort, imaginer très exactement les circonstances environnantes, les fleurs, les cierges, l'odeur funèbre, les lèvres pâlies et les paupières mal closes sur les yeux révulsés. [...] Je renouvelais ce fantasme sans y rien modifier, toutes les fois que mon désir l'exigea et il me procura pendant longtemps des voluptés extrêmement vives (Wittkop, 2001, p. 31-32).

Le concept de fantasme est lié à celui de libido tel qu'il est présenté chez Freud. Comme l'incestueux est incapable de reporter ses désirs sexuels sur un objet étranger, il est impossible pour le sujet nécrophile de déplacer ses investissements libidineux sur un objet vivant. Ainsi, l'énergie pulsionnelle accumulée par le fantasme n'est libérée qu'au moment du passage à l'acte.

\section{LA REPRÉSENTATION DE LA NÉCROPHILIE OU LE PASSAGE À L'ACTE}

Le personnage du nécrophile ne s'en tient pas, en effet, qu'à la représentation fantasmatique de son désir. Progressivement, il transgresse les frontières de l'interdit lié à la mort en inhalant l'air ambiant des cadavres 
gisant dans son appartement. Qui plus est, l'odeur de chair en décomposition n'inspire pas de répugnance au nécrophile; plutôt que de s'en éloigner, il s'en imprègne parce qu'il la trouve agréable. De sa mère, il dit: «[...] je respirai son odeur enivrante» (Wittkop, 2001, p. 17), expression qui témoigne sans équivoque de l'amour du personnage pour le corps inanimé de celle qui lui a donné la vie. Et que dire de Suzanne, cette dépouille de femme qui devient le plus grand amour de sa vie? Installée depuis déjà plusieurs jours dans son appartement, elle dégage une odeur nauséabonde au milieu de laquelle il n'éprouve aucune difficulté à trouver le sommeil: «Rentré chez moi, je me jetai sur un lit qui sentait la charogne. Je m'endormis d'un seul coup, brutalement saisi par un sommeil mortel, bercé par les mêmes flots noirs - mare tenebrarum - qui berçaient Suzanne, Suzanne mon amour. » (Wittkop, 2001, p. 44.)

Outre le fait que le nécrophile partage son appartement avec des cadavres, ce personnage repousse encore plus loin les barrières de l'interdit en manipulant concrètement les morts; c'est là que nous pouvons dire qu'il transgresse véritablement le tabou. Pour cela, nous nous rapportons ici à la définition même du mot "tabou», soit ce qui est sacré, ce qu'il ne faut pas toucher sans commettre un sacrilège (Robert, 1992, p. 2072). En effet, non seulement le nécrophile entre-t-il directement en contact avec les dépouilles, mais il s'applique également à leur attribuer des traits anthropomorphes en leur faisant poser des gestes propres aux vivants; une attitude qu'on pourrait qualifier d'animiste. À cet effet, il serait fort intéressant de tenter de rapprocher, d'un point de vue sémiotique, cette tendance à considérer le mort vivant avec l'attitude mélancolique. Cette dernière se caractérise non seulement par un déni de la mort (anthropologique) d'autrui mais par un refus d'abandon de ses restes. Autrement dit, le sujet nécrophile tel qu'il est représenté dans ce roman ne réussirait pas véritablement à tuer l'autre. N'étant pas parvenu à faire le deuil que suppose la perte de l'objet d'amour, Lucien s'acharne sur la dépouille d'autrui en tentant d'agir comme si rien n'avait changé. Or, pour le nécrophile de même que pour le mélancolique, il est impossible de panser la perte ${ }^{6}$.

Le contact physique s'intensifiant, le lecteur assiste à des scènes où le nécrophile prend soin de laver les cadavres. Et de ce fait, le protagoniste se trouve à entretenir un réel contact avec le corps inanimé de l'autre. Par exemple, le dernier soir où Henri partage la vie de Lucien, ce dernier s'affaire à lui donner son bain: "Je l'ai séché dans un drap de bain, je lui ai remis le petit pyjama de finette bleue qu'il

POUR ASSOUVIR SON DÉSIR DE VIE (ÉROS),

LE SUJET NÉCROPHILE FAIT APPEL

\section{À SES PULSIONS DE MORT (THANATOS).}

portait en arrivant, j'ai lissé ses franges brunes que l'eau du bain faisait paraître presque noires. Dans la voiture, je l'avais assis près de moi, le soutenant d'une main, conduisant de l'autre» (Wittkop, 2001, p. 34). Que ce soit par pure coïncidence ou encore par une volonté consciente de Gabrielle Wittkop d'introduire dans cette transgression un certain symbolisme relatif au pouvoir de l'eau en tant qu'élément ${ }^{7}$ à la fois purificateur et destructeur du tabou qui pèse sur l'acte nécrophile, le narrateur ne procède pas à ce rituel qu'une seule fois dans le roman. Il accentue l'effet produit en répétant le geste une seconde fois avec la dépouille de Suzanne: "Le dernier soir, j'ai lavé Suzanne, je lui ai remis son linge fin $[. .$.$] que deux semaines plus tôt$ je lui avais retiré dans l'euphorie. Entourée d'un plaid, je l'ai portée jusqu'à la voiture»(Wittkop, 2001, p. 44). On voit ainsi que les cas d'Henri et de Suzanne se ressemblent car avant de se départir de chacun de ces deux corps, le nécrophile prend soin de les nettoyer comme s'il voulait effacer toute trace du crime. Ensuite, il les installe sur le siège de sa voiture où il les reconduit à leur ultime demeure: la Seine. Serait-ce là le témoignage d'un besoin intrinsèque de purification soustendu par une conscience coupable envers la transgression dont le nécrophile est l'auteur?

Outre les bains que le protagoniste donne à ses cadavres, d'autres situations imposent une intimité physique d'autant plus grande comme lorsqu'il embrasse Suzanne dans le cimetière et qu'il passe la nuit avec Marie-Jeanne Chaulard:

[...] je me suis couché cette nuit à côté d'elle, pour dormir quelques heures, comme l'époux près de l'épouse, un bras passé sous la nuque mince, une main posée sur le ventre où j'avais trouvé quelque joie. [...] Je caresse légèrement les cheveux gris et maigres, tirés en arrière, le cou et les épaules [...]

(Wittkop, 2001, p. 29).

Cette proximité avec la dépouille d'une personne âgée relève d'une certaine transgression au sein des sociétés occidentales parce que son atteinte est double: non seulement elle transgresse l'interdit lié à la mort, mais en plus, elle montre les aînés comme des êtres sexués, chose qu'on a souvent tendance à oblitérer.

\section{LA MISE EN SCÈNE DE L'ACTE SEXUEL: UNE SUBVERSION DU RAPPORT À LA MORT}

Le personnage du nécrophile assujetti à son désir d'assouvissement de ses pulsions érotiques poursuit sa quête de l'autre (qui n'est plus, tel est le paradoxe interrogé ici) en parvenant à ce qu'on pourrait considérer comme le paroxysme de l'intimité physique. Le sujet ne se contente pas de manipuler, d'embrasser, de caresser ses cadavres, mais il copule avec eux. Selon Germain et Langis, le protagoniste de ce roman représenterait la forme la plus avancée de nécrophilie; il déterre lui-même les corps fraîchement inhumés avant de se livrer à des activités sexuelles sur eux. Toutefois, il est impossible pour ces psychologues de le qualifier de "psychopathe sadique» (Germain et Langlois, 1990, p. 327) parce qu'il ne tue pas lui-même ses victimes avant de s'en servir.

Nombreuses sont les dépouilles avec lesquelles le nécrophile accomplit charnellement l'objet de ses «passions» (Sade, 1975) dont deux d'entre elles sont vierges: la demoiselle d'Ivry et Teresa. Il nous semble tout à fait pertinent de devoir attirer ici l'attention sur ce qui se révèle être, dans ce contexte, un élément doublement tabou. Il s'agit de la perte de virginité qui renforce le caractère transgressif de la relation sexuelle comme le souligne Bataille: "L'acte sexuel a toujours une valeur de forfait, dans le mariage, hors du mariage. Il l'a surtout s'il s'agit d'une vierge: il l'a toujours un peu la première fois » (Bataille, 2001, p. 122). C'est aussi ce que constate Freud dans une étude intitulée Le tabou de la virginité: "Le premier rapport sexuel est certes un acte lourd de conséquences; il l'est d'autant plus si le sang doit y couler»(Freud, 1970, p. 71). Il va même jusqu'à ajouter que «ce n'est pas seulement le premier coït avec la femme qui est tabou: tous les rapports sexuels le sont»(Freud, 1970, p. 71). Dans cet essai, le psychanalyste relate l'importance chez les peuples primitifs de la défloration. Le tabou de la virginité n'ayant pas complètement disparu de nos sociétés contemporaines, découle en dernière instance de cette crainte du sang associée, dans la pensée collective, à l'interdit du meurtre et, par extension, à celui de la mort. Alors, que dire de la défloraison d'une dépouille?

Donc, sur un total de seize cadavres mis en scène dans ce roman, la mère du nécrophile, Marie-Jeanne Chaulard, la Nonne 
et Pierre sont les seuls personnages avec lesquels le protagoniste ne se livre pas à des pratiques sexuelles. Toutefois, avec Suzanne l'acte charnel prend un tout autre sens. Avec elle, le nécrophile accède à une union qui engage tout son être:

Je portai Suzanne sur mon lit. D'une main tremblante, je lui enlevai son soutien-gorge, sa petite culotte. [...] Je me jetai sur cette morte charmante et sans même la débarrasser de son porte-jarretelles ni de ses bas, je la pris avec une ferveur et une violence que je n'avais, je crois, jamais éprouvées jusqu'alors. [...] Je la couvrais de caresses, je léchais tendrement son sexe, je le broutais avec avidité, je m'y plongeais et m'y replongeais sans cesse, lorsque je ne préférais pas les délices de Sodome

(Wittkop, 2001, p. 40-42).
Juan de Molière, n'entretient des liens privilégiés qu'avec une seule femme à la fois, ce qui tend à assouplir le caractère transgressif de l'acte lui-même.

De plus, bien que la société occidentale semble de moins en moins ostraciser l'homosexualité, cette dernière demeure quelque chose que le catholicisme n'est pas le seul à enfouir dans la fosse aux tabous. Admettant cette hypothèse, le personnage du nécrophile accentuerait la gravité de son sacrilège en n'ayant pas uniquement des relations sexuelles avec des femmes mais aussi avec trois individus de sexe masculin: Jérôme, Henri et le Suédois.

On ne pourrait en outre passer sous silence l'interdit de la pédophilie qui constitue l'un des tabous majeurs en Occident. C'est dans cette perspective qu'il nous est possible d'affirmer que le texte de Gabrielle Wittkop transgresse «la loi du silence»

POUR LE DIRE D'UNE FAÇON UN PEU BRUTALE, LA SOCIÉTÉ CONTEMPORAINE SE NOURRIT DES DÉPOUILLES D'AUTRUI.

\section{L'INTERDIT DE LA MORT} COMME MISE EN ABYME

Le personnage du nécrophile transgresse les règles sociales en cultivant des relations intimes avec les cadavres, mais il soulève également dans ses pratiques d'autres interdits, ce qui amplifie le caractère subversif de l'écriture. C'est dans une sorte de mise en abyme que sont représentés les tabous. Tout comme Eugénie de Mistival élève de La Philosophie dans le boudoir affirmant être «à la fois incestueuse, adultère, sodomite, et tout cela pour une fille qui n'est dépucelée que d'aujourd'hui » (Sade, 1976, p. 278) ${ }^{8}$, le nécrophile de Gabrielle Wittkop n'échappe pas à cette pluralité. La nécrophilie permet ici à d'autres tabous de s'affranchir, c'est-à-dire de perdre une parcelle de leur identité originelle pour devenir subversion. La promiscuité exacerbée ${ }^{9}$ serait alors à inscrire dans cette optique puisque comme le veut la société occidentale, l'homme se doit de n'être fidèle qu'à une seule femme, sa conjointe (après avoir ou non convolé en justes noces). Mais dans ce roman, le protagoniste (célibataire) cumule, non pas les rencontres, mais les partenaires dans une période de temps relativement courte (environ un an au total). Parmi ses conquêtes, on compte la petite fille-pieuvre, la Suédoise, l'actrice, Gabrielle, Suzanne, la demoiselle d'Ivry, Geneviève ainsi que la naine Teresa. Il importe toutefois de dire que le nécrophile, contrairement au Dom quand il met en scène un protagoniste qui, en plus d'être nécrophile, s'avère pédophile. Le nécrophile qui nous est présenté assouvit ses pulsions sexuelles avec quatre enfants soit, la petite fille-pieuvre, Henri, Jérôme (15 ans) ainsi que l'enfant mortné de Geneviève avec qui il ne se refuse aucunement l'accès: «Je m'introduisis simplement entre ses cuisses mais pour bientôt m'apercevoir que je n'en tirerais nul plaisir» (Wittkop, 2001, p. 66). Néanmoins, à l'opposé du pédophile invétéré, il est impossible d'affirmer que le narrateur éprouve de réel plaisir à cette pratique. Ce qui l'intéresse avant tout, c'est d'assouvir ses fantasmes avec des morts matures et non pas avec des enfants considérant le discours qu'il tient à ce propos.

L'analyse ne saurait pas être complète si nous nous abstenions d'évoquer que cette parole littéraire lève le voile sur un autre tabou: l'inceste. Ce n'est pas que le protagoniste entretienne des relations charnelles avec un membre de sa famille, mais qu'il assouvit certains fantasmes en donnant forme à un acte incestueux de nature fraternelle entre les Suédois (le frère et la sœur) :

Je les ai couchés dans les bras l'un de l'autre, les enlaçant tendrement, posant les lèvres du frère sur celles de la sœur, engageant le sexe endormi de l'un entre les nymphes délicates de l'autre [...] J'ai voulu que leurs corps qui, dans la vie, avaient dû si souvent s'appeler en secret, s'unissent enfin dans la mort (Wittkop, 2001, p. 91).

Enfin, Le Nécrophile de Gabrielle Wittkop relève d'une écriture «métatopique» puisqu'il parvient à représenter le rapport à la mort dans une perspective de transgression visant à remettre en question la parole clichée. La cohabitation avec les dépouilles livre des savoirs d' «outre-tombe ${ }^{10}$ » grâce auxquels le stéréotype de la mort est subverti. Loin de faire appel à une esthétique grotesque, ce roman préconise une écriture du raffinement. Le sujet nécrophile y est représenté dans son exclusion, c'est-à-dire à partir d'une tentative de représentation de la vie dans la mort où les dépouilles en acquérant un statut onomastique reviennent interagir avec les vivants. Les cadavres n'y sont ni bleus ni froids comme on a trop souvent tendance à les imaginer mais ils sont, au contraire, représentés comme des êtres vivants. C'est dire que les morts sont récupérés du dépotoir anonyme dans lequel ils baignent pour, en quelque sorte, se réincarner; une fois que le protagoniste les déterre, il leur confère une nouvelle identité. Ainsi, l'ordre habituel des choses se trouve inversé par la mise en scène de la nécrophilie qui se conjugue, dans une sorte de mise en abyme, à plusieurs autres interdits sexuels représentant un écart par rapport à la norme: la promiscuité exacerbée, l'homosexualité, la pédophilie et l'inceste; la preuve qu'une transgression ne se manifeste pas dans sa singularité. En dernière analyse, la représentation de la sexualité du nécrophile telle qu'elle se dégage du roman de Wittkop peut-elle être pensée comme la rencontre du même et de l'autre ou faut-il, à l'inverse, croire qu'il s'agit là d'un rapport d'altérité miné à la base? Dans ce contexte, quelles seraient les conditions d'émergence pour une représentation du rapport à la mort où l'autre, tout comme le même, ne serait pas l'objet de quelque forme d'exclusion?

\section{Bibliographie}

BACHAND, Denis (2005). "Mourir à l'écran: entre réalité et simulation », Frontières, vol. 18, $\mathrm{n}^{\mathrm{o}} 1$, automne, p. 59.

BATAILLE, Georges (2001). L'Érotisme, $2^{\text {e }}$ éd., Paris, Les Éditions de Minuit, coll. «Arguments».

BATAILLE, Georges (1973). Madame Edwarda, Paris, 10/18, coll. "Domaine français».

BIBLIOROM LAROUSSE (1996), version Windows, [Cédérom], Paris, Larousse, Microsoft Corporation.

CASTILLO DURANTE, Daniel (1994). Du stéréotype à la littérature, Montréal, XYZ, coll.«Théorie et littérature».

CASTILLO DURANTE, Daniel (2004). Les dépouilles de l'altérité, Montréal, XYZ, coll.«Documents». 
CHATEAUBRIAND, François-René de (1948). Mémoires d'outre-tombe, Paris, Flammarion, coll. «Les Grands mémoires» [1849].

DELORME, Julie (2005). «Putain, stéréotype ou identité en marge: Putain de Nelly Arcan ", Les discours économique et symboliques d'exclusion et d'inclusion: perspectives américaines, dir. Daniel Castillo Durante, Amy Colin et Patrick Imbert, Ottawa, Legas, «Collection des Amériques», p. 241-252.

FREUD, Sigmund (1970). «Le tabou de la virginité », La vie sexuelle, $2^{\mathrm{e}}$ éd., trad. par Denise Berger, John Laplanche et al., Paris, Presses universitaires de France.

GERMAIN, Bernard, et Pierre LANGIS (1990). La sexualité: regards actuels, préf. de Gilbert Tordjman et Claude Crépault, Laval, Études vivantes.

MILAT, Christian (2005). "L'alchimie, ou la mort transmutée en immortalité », Frontières, vol. $18, \mathrm{n}^{\circ} 1$, p. 33.

MORIN, Pierre-Charles et Suzanne BOUCHARD (1997). Introduction aux théories de la personnalité, $2^{\mathrm{e}}$ éd., Montréal, Gaëtan Morin.

ROBERT, Paul (2004). Le nouveau petit Robert dictionnaire de la langue française 1 , Paris, Le Robert.

ROBERT, Paul (1992). Le Robert dictionnaire historique de la langue française, dir. Alain Rey, Paris, Le Robert.

SADE, Le marquis de (1987). Histoire de Juliette, ou les Prospérités du vice, Paris, Pauvert.
SADE, Le marquis de (1976). La Philosophie dans le boudoir, éd. présentée, établie et annotée par Yvon Belaval, Paris, Gallimard, coll. «Folio classique».

SADE, Le marquis de (1975). Les 120 Journées de Sodome, préf. de Gilbert Lely, Paris, 10/18, coll. «Domaine français».

WITTKOP, Gabrielle (2001). Le Nécrophile, $4^{\mathrm{e}}$ éd., Paris, Verticales/Le Seuil.

\section{Notes}

1. Georg Wilhelm Friedrich Hegel cité par Georges Bataille en exergue dans la préface de Madame Edwarda, Paris, 10/18, coll. «Domaine français», 1973, p. 11.

2. Voir à ce sujet l'article de Denis Bachand dans ce numéro: "Mourir à l'écran: entre réalité et simulation».

3. Il semble pertinent de rappeler ici l'enjeu majeur à l'origine du mythe de Psyché soit celui de la transgression. Un soir Psyché, une belle jeune fille aimée d'Éros, désobéit à Zeus lui ayant interdit de voir le visage de son amant en allumant une lampe. À la suite de cet incident (manifestation d'une curiosité exacerbée), Éros s'enfuit et disparaît pour ne retrouver Psyché qu'au terme d'une longue suite d'aventures. Cf. Bibliorom Larousse, version Windows, [Cédérom], Paris, Larousse, Microsoft Corporation, 1996.

4. Voir à ce propos l'article de Christian Milat dans ce numéro: «L'alchimie, ou la mort transmutée en immortalité» dans lequel le «Grand Euvre» apparaît comme l'expression d'une atemporalité illustrée par la figure de l'oroboros («le serpent qui se mord la queue»).
5. Au sens où l'entend Freud.

6. Il est possible d'établir un rapprochement entre la mélancolie et la nécrophilie dans la mesure où elles se découvrent en fonction d'une perte. Le mélancolique, comme le nécrophile, est incapable de faire le deuil du passé; son existence est fondée sur un désir d'antériorité. Pour une analyse plus approfondie du concept de mélancolie se référer à l'ouvrage de Daniel Castillo Durante, Les dépouilles de l'altérité, op. cit.

7. Dans ce contexte, l'eau fait partie des quatre éléments de la cosmologie traditionnelle avec l'air, la terre et le feu.

8. Il paraît intéressant de faire remarquer ici que Sade semble avoir tendance à faire de ses héros romanesques des transgresseurs de plusieurs interdits à la fois. Outre Eugénie, c'est aussi le cas du ministre Saint-Fond: "J'étais couverte de malédictions, d'imprécations, je parricidais, j'incestais, $j$ 'assassinais, je prostituais, je sodomisais ", Histoire de Juliette, ou les Prospérités du vice, Paris, Pauvert, 1987, p. 292.

9. Faute de mieux, nous voulons par cette expression désigner le sujet qui accumule de nombreux partenaires avec qui il n'est pas uni par les liens sacrés du mariage. La «promiscuité exacerbée» remplacerait, dans le contexte des unions de fait, les termes «polygamie» et «polyandrie» relatifs aux unions dites légitimes.

10. Cette expression n'est pas sans faire allusion au célèbre titre de François-René de Chateaubriand: Mémoires d'outre-tombe (1849). 